\title{
Evaluation of caregiver burden of cancer patients and impact of this burden on caregiver's quality of life
}

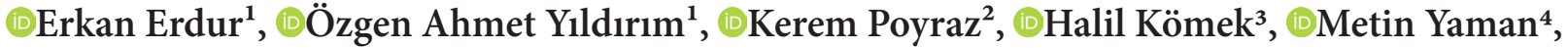 \\ ๑Mustafa Çelik ${ }^{5}$ \\ Gazi Yaşargil Training and Research Hospital, Department of Internal Medicine, Division of Medical Oncology, Diyarbakır, Turkey \\ Gazi Yaşargil Training and Research Hospital, Department of Radiation Oncology, Diyarbakır, Turkey \\ Gazi Yașargil Training and Research Hospital, Department of Nuclear Medicine, Diyarbakır, Turkey \\ Gazi Yaşargil Training and Research Hospital, Department of Internal Medicine, Diyarbakır, Turkey \\ Gazi Yaşargil Training and Research Hospital, Department of Psychiatry, Diyarbakır, Turkey
}

Cite this article as: Erdur E, Yıldırım ÖA, Poyraz K, Kömek H, Yaman M, Çelik M. Evaluation of caregiver burden of cancer patients and impact of this burden on caregiver's quality of life. J Health Sci Med 2022; 5(1): 313-320.

\begin{abstract}
Aim: To predict the caregiver burden based on the clinical characteristics of cancer patients and the social and economic circumstances of the caregiver, and to evaluate the influence of this burden on the caregiver's quality of life.

Material and Method: The effects of the clinical findings of 411 patients followed up and treated in our clinic between January 2020 and March 2021, and the social and economic circumstances of the caregiver on the Zarit caregiver burden questionnaire score filled by the caregivers were analyzed. In addition, the Zarit caregiver burden score obtained was researched how influenced the Short-form 36 quality of life questionnaire filled by the caregivers.

Results: In our study, a statistically significant correlation was found between Zarit score and ECOG PS, transportation, residence status, receiving chemotherapy, having a metastatic disease ( $\mathrm{p}<0.05$ for all) in linear regression analysis of Zarit score with pairwise and more than two groups analysis. A moderate reverse relationship was determined between the Zarit scores of caregivers and SF36 scores ( $\mathrm{p}<0.05$ for all).

Conclusion: The caregiver burden is affected by the patient's clinical characteristics and the social and economic circumstances of the caregiver. Increasing the caregiver burden has adverse effects on the caregiver's quality of life. These findings show the importance of considering the caregiver factor in the evaluation of treatment compliance and well-being of patients.
\end{abstract}

Keywords: Cancer, caregiver burden, zarit caregiver burden questionnaire, short form 36

\section{INTRODUCTION}

Approximately 19.3 million new cancer cases were diagnosed globally in 2020; meanwhile, 10 million cancer patients died due to this disease (1). Cancer, responsible for one out of every six deaths globally, is the second most common cause of death after cardiovascular-related deaths (2). Despite today's contemporary diagnosis and treatment protocols, the potentially fatal cancer disease and its treatment have devastating effects on patients physically and psychologically.

Relatives, spouses, and close friends who have a meaningful personal relationship with the patient, take care of the cancer patient, and provide a wide range of help are also affected by the psychological and physical trauma of the cancer disease (3).
Patients with a diagnosis of cancer often require direct support by a caregiver to assist with activities of daily living, administer medications, provide transportation, prepare meals, manage finances, sustaining medical care, and provide emotional support. Caregiving is hard work. About a quarter of the caregivers of cancer patients spend more than 40 hours in a week providing this support to their family or friends (4).

Increasing day by day, more evidence demonstrates that individuals exposed to caregiver burden experience psychological, behavioral, and physiological effects that may contribute to impaired immune system function, coronary heart disease, and premature death compared to non-caregivers (5-6). Therefore, caregivers of cancer patients remain under both psychological and physical 
stress and have a lower quality of life by comparison with non-caregivers. Furthermore, caregiver burden and quality of life (QoL) have been investigated in various diseases, including dialysis and transplant recipients, chronic kidney disease, schizophrenia, Parkinson's disease, inflammatory bowel disease, and Alzheimer's disease (7-10). So, we aimed to evaluate the caregiver burden, quality of life, and health perception of the caregiver based on the clinical characteristics of the patients and the social and economic conditions of the caregiver in this study. Providing a systematic quality of life analysis and caregiver burden monitoring of the caregiver of cancer patients was intended with the results we obtained. In addition to this, we aimed to form a referrer guide about the rational and practical distribution of social services provided to patients and their caregivers during cancer treatment has an escalating economic burden.

\section{MATERIAL AND METHOD}

This study received approval from the Institutional Review Board of Health Sciences University Diyarbakır Gazi Yaşargil Training and Research Hospital Ethics Committee (Date 09.06.2021; Decision No: 829). All procedures were carried out in accordance with the ethical rules and the principles of the Declaration of Helsinki.

Primary caregivers of 468 patients with a performance score of 3 or less, followed up for at least three months in Diyarbakır Gazi Yaşargil Training and Research Hospital Medical Oncology Polyclinics between January 2020 and March 2021, were included in this descriptive cross-sectional study. The clinical characteristics of the patients were recorded considering the examination in the last outpatient visit, and a medical oncology specialist evaluated the findings. Zarit Caregiver Burden Interview (ZCBI) and Short Form 36 (SF-36) were filled in by the primary caregivers of the patients in the same conditions in the medical oncology meeting room. All caregivers who were the primary caregivers of a patient diagnosed with cancer and were older than 18 years of age and could read and write in Turkish without significant comorbidity or limitation of the movement were included in the study. 57 of 468 primary caregivers were excluded from the study because of uncontrolled diabetes, uncontrolled hypertension, cardiovascular and cerebrovascular disease, language problems, and purchasing care services. 411 patients and their primary caregivers were evaluated.

\section{Data Collection and Questionnaires}

Zarit Caregiver Burden Interview (ZCBI) and Short Form 36 were used as data collection tools.

1. Zarit caregiver burden interview (ZCBI): ZCBI was developed by Zarit, Reever, and Bach Peterson in 1980
(11). The questionnaire, which was originally designed with 29 questions, was then revised as a questionnaire of 22 questions. It is a scale used to evaluate the stress and psychological well-being of caregivers. The scale, which can be filled in by the caregivers themselves or by the researcher, determines the impact of caregiving on the individual's life. Turkish-approved ZCBI was published in 2009 by Ozlu et al. The Turkish version consists of 19 items. Each item is scored from 1 to 5 as $1=$ never, $=$ rarely, $3=$ sometimes, $4=$ quite often, and $5=$ almost always. Caregiver burden is assessed according to the total score obtained from all responses. Total scores are calculated between 19 and 95 points, and a higher score indicates a higher caregiver burden. A score of 21 or less demonstrates without burden, 22-46 having a light burden, 47-55 having a moderate burden, and 56 or more having a severe load(12).

2. Short form 36: Short Form 36, which provides wideangle measurement within the quality of life scales, was developed and made available by Rand Corporation in 1992. The form, which was designed as 149 questions in the development phase, was simplified and then converted into a short form as 36 questions by adding psychometric features. The scale consists of 36 items. The questions provide measuring eight dimensions. Physical function, social function, role limitations due to physical functions, role limitations due to emotional problems, mental health, energy/vitality, pain, and general perception of health are evaluated by the scale. Instead of giving only a single total score, the scale provides a total score for each subscale separately. Subscales peruse health between 0 and 100, so 0 expressing poor health, 100 expressing good health (13-14). The reliability and validity study of the Turkish version of SF-36 was performed by Koçyiğit et al. (15).

\section{Stratification Factors}

1.Patient-related factors: Patient-related factors were determined as patient age, gender, educational status, comorbidities, cancer type, active chemotherapy treatment, whether or organ metastasis.

2.Caregiver related factors: Caregiver age, education level, intimacy with the patient (spouse, child, sibling, other relatives, friend), comorbidities were evaluated as factors related to the caregiver.

3. Economic conditions: Factors related to the economic status of the caregiver were determined as the location of his home (city center, district, village), whether the caregiver owning a vehicle, the caregiver owned or rented a house.

\section{Statistical Method}

SPSS (Statistical Package for the Social Sciences) 23.0 package program was used for statistical analysis of the 
data. Categorical measurements were stated as numbers and percentages, and continuous measurements were expressed as mean and standard deviation (median and minimum-maximum where appropriate). Chisquare and Fisher exact certainty diagnostic tests were used to compare categorical parameters. In addition, the Shapiro-Wilk test was used to determine whether or the parameters in the study normally distributed. Mann-Whitney test was used in pairwise comparisons for parameters that did not normally distribute, and the Kruskal Wallis test was used in more than two group analyses. Finally, Spearman correlation tests were used to specify the relationship between Zarit score and SF 36 scale scores. A multiple linear regression model was used to identify the factors affecting the Zarit score. Statistical significance level was acknowledged as 0.05 in all tests.

\section{RESULTS}

The characteristics of patients and caregivers are summarized in Table 1. In evaluating the caregiver's Zarit score and the patient's PS ( $\mathrm{p}<0.05$ for all), the caregivers of PS: 1 group patients compared to PS: 0 group patients were found to have a higher Zarit caregiver burden score. The caregivers of PS: 2 group patients compared to PS: 0 and PS: 1 groups were found to have a higher Zarit caregiver burden mean. The caregivers of PS: 3 group patients compared to PS: 0, PS:1 and PS:2 groups were found to have a higher Zarit caregiver burden mean.

\begin{tabular}{|c|c|c|}
\hline & Frequency (n) & Percent (\%) \\
\hline \multicolumn{3}{|l|}{ Patients Gender } \\
\hline Female & 172 & 41.8 \\
\hline Male & 239 & 58.2 \\
\hline \multicolumn{3}{|l|}{ Cancer Type } \\
\hline Lung & 65 & 15.8 \\
\hline Bladder & 6 & 1.5 \\
\hline Thyroid & 31 & 7.5 \\
\hline Melanoma & 8 & 1.9 \\
\hline Pancreas & 10 & 2.4 \\
\hline Brain & 11 & 2.7 \\
\hline Testis & 4 & 1.0 \\
\hline Sarcoma & 9 & 2.2 \\
\hline Head and Neck & 9 & 2.2 \\
\hline Others & 30 & 7.3 \\
\hline Breast & 51 & 12.4 \\
\hline Prostate & 43 & 10.5 \\
\hline Colorectal & 58 & 14.1 \\
\hline Stomach & 39 & 9.5 \\
\hline Kidney & 10 & 2.4 \\
\hline Uterine & 8 & 1.9 \\
\hline Cervix & 7 & 1.7 \\
\hline Ovarian & 12 & 2.9 \\
\hline \multicolumn{3}{|l|}{ Patient Comorbidity } \\
\hline Unavailable & 224 & 54.5 \\
\hline
\end{tabular}

\begin{tabular}{|c|c|c|}
\hline Available & 187 & 45.5 \\
\hline \multicolumn{3}{|l|}{ Ecog PS } \\
\hline 0 & 13 & 3.2 \\
\hline 1 & 177 & 43.1 \\
\hline 2 & 172 & 41.8 \\
\hline 3 & 49 & 11.9 \\
\hline \multicolumn{3}{|l|}{ Organ Metastasis } \\
\hline Unavailable & 261 & 63.5 \\
\hline Available & 150 & 36.5 \\
\hline \multicolumn{3}{|l|}{ Chemotherapy } \\
\hline Unavailable & 247 & 60.1 \\
\hline Available & 164 & 39.9 \\
\hline \multicolumn{3}{|l|}{ Patients Education } \\
\hline Illiterate & 19 & 4.6 \\
\hline Literate & 121 & 29.4 \\
\hline Primary School Education & 162 & 39.4 \\
\hline High School and Above & 109 & 26.5 \\
\hline \multicolumn{3}{|l|}{ Caregiver transportation } \\
\hline Public Transport & 169 & 41.1 \\
\hline Special Vehicle & 242 & 58.9 \\
\hline \multicolumn{3}{|l|}{ Caregiver Place of Residence } \\
\hline Village, District & 162 & 39.4 \\
\hline City Centre & 249 & 60.6 \\
\hline \multicolumn{3}{|l|}{ Cargiver Residence Status } \\
\hline Tenant & 159 & 38.7 \\
\hline Own House & 252 & 61.3 \\
\hline \multicolumn{3}{|l|}{ Caregiver İntimacy } \\
\hline Spouse & 200 & 48.7 \\
\hline Son & 95 & 23.1 \\
\hline Sibling & 53 & 12.9 \\
\hline Relative & 63 & 15.3 \\
\hline \multicolumn{3}{|l|}{ Caregiver Gender } \\
\hline Female & 208 & 50.6 \\
\hline Male & 203 & 49.4 \\
\hline \multicolumn{3}{|l|}{ Caregiver Education } \\
\hline Literate & 108 & 26.2 \\
\hline Primary School Education & 182 & 44.3 \\
\hline High School and Above & 121 & 29.5 \\
\hline \multicolumn{3}{|l|}{ Caregiver Comorbidity } \\
\hline Unavailable & 274 & 66.7 \\
\hline Available & 137 & 33.3 \\
\hline \multicolumn{3}{|l|}{ Zarit Carrgiver Load Weight } \\
\hline None & 9 & 2.2 \\
\hline Light Load & 188 & 45.7 \\
\hline Medium Load & 111 & 27.0 \\
\hline \multirow[t]{2}{*}{ Heavy Load } & 103 & 25.1 \\
\hline & Mean \pm sd & Med (Min-Max) \\
\hline Patients Age & $58.4 \pm 13.5$ & $61(21-92)$ \\
\hline Caregivers Age & $51.3 \pm 10.6$ & $53(24-70)$ \\
\hline Zarit Score & $46.7 \pm 14.0$ & $48(20-79)$ \\
\hline SF36 Physical function & $45.9 \pm 10.8$ & $48(20-77)$ \\
\hline SF36 Physical Role Difficulty & $45.4 \pm 11.0$ & $47(12-69)$ \\
\hline SF36 Pain & $46.7 \pm 9.0$ & $48(22-67)$ \\
\hline SF36 General Health & $47.6 \pm 8.9$ & $48(24-70)$ \\
\hline SF36 Energy /Vitality & $45.6 \pm 7.9$ & $46(23-73)$ \\
\hline SF36 Social Functionality & $53.5 \pm 9.2$ & $54(24-77)$ \\
\hline SF36 Emotional Role Difficulty & $48.8 \pm 11.6$ & $51(20-71)$ \\
\hline SF36 Mental Healt & $52.3 \pm 8.6$ & $52(24-70)$ \\
\hline
\end{tabular}


It was determined that the Zarit score differed according to the patient's education level. It was understood that the difference was due to those with a high school or higher graduation had lower scores than those who uneducated, literate, and had primary education $(\mathrm{p}<0.05$ for all). There was no significant difference between the other groups ( $p>0,05$ for all).

There was no significant relationship between caregiver education level and Zarit caregiver burden $(\mathrm{p}=0.347)$.

In addition, the caregivers who looked after patients who aged 61 and above, male, with additional comorbidities, with organ metastases, receiving chemotherapy and the caregivers who used public transportation, dwelt in villages or towns, became tenants, had comorbidities, were male were found to have higher Zarit scores $(\mathrm{p}<0.005$ for all)

According to Bonferroni \& Tamhane's test, which was performed to detect the cause of the significant difference in the assessment of the Zarit score in terms of the intimacy between patient and caregiver, it was determined that the caregiver who was the patient's child had a higher score than the spouse who was the caregiver $(\mathrm{p}<0.05)$. (Table 2)

\begin{tabular}{|c|c|c|c|c|c|}
\hline \multicolumn{6}{|c|}{ Zarit } \\
\hline & & Med (Min-Max) & & & Med (Min-Max) \\
\hline \multicolumn{2}{|l|}{ Patients Gender } & & \multicolumn{3}{|l|}{ Cargiver Residence Status } \\
\hline Female & & $42(20-75)$ & Rent & & $51(20-79)$ \\
\hline \multirow[t]{3}{*}{ Male } & & $51(20-79)$ & Own House & & $42(20-78)$ \\
\hline & $\mathrm{u}$ & $-5,947$ & & $\mathrm{u}$ & $-6,024$ \\
\hline & $\mathrm{p}$ & $<0,001$ & & $\mathrm{p}$ & $<0,001$ \\
\hline Age & & & Caregiver Gender & & \\
\hline $61<$ & & $48(20-79)$ & Female & & $45(20-79)$ \\
\hline \multirow[t]{3}{*}{$61 \geq$} & & $47(20-78)$ & Male & & $48(20-78)$ \\
\hline & $\mathrm{u}$ & $-3,017$ & & $\mathrm{u}$ & $-6,024$ \\
\hline & $\mathrm{p}$ & 0,003 & & $\mathrm{p}$ & $<0,001$ \\
\hline PatientComorbidity & & & Caregiver Comorbidity & & \\
\hline Unavailable & & $43(20-77)$ & Unavailable & & $44(20-78)$ \\
\hline \multirow[t]{3}{*}{ Available } & & $50(20-79)$ & Available & & $51(20-79)$ \\
\hline & $\mathrm{u}$ & $-5,514$ & & $\mathrm{u}$ & $-5,055$ \\
\hline & $\mathrm{p}$ & $<0,001$ & & $\mathrm{p}$ & $<0,001$ \\
\hline Organ Metastasis & & & Caregiver Place of Residence & & \\
\hline Unavailable & & $38(20-76)$ & Village, County & & $50,5(20-79)$ \\
\hline \multirow[t]{3}{*}{ Available } & & $58(33-79)$ & Town Centre & & $43(20-77)$ \\
\hline & $\mathrm{u}$ & $-13,220$ & & $\mathrm{u}$ & $-5,366$ \\
\hline & $\mathrm{p}$ & $<0,001$ & & $\mathrm{p}$ & $<0,001$ \\
\hline Chemotherapy & & & Caregiver Transportation & & \\
\hline Unavailable & & $38(20-78)$ & Public Transport & & $52(20-79)$ \\
\hline \multirow[t]{3}{*}{ Available } & & $54,5(22-79)$ & Special Vehicle & & $41(20-78)$ \\
\hline & $\mathrm{u}$ & $-12,051$ & & $\mathrm{u}$ & $-7,316$ \\
\hline & $\mathrm{p}$ & $<0,001$ & & $\mathrm{p}$ & $<0,001$ \\
\hline ECOG PS & & & Caregiver İntimacy & & \\
\hline 0 (a) & & $22(20-44)$ & Spouse (a) & & $43(20-78)$ \\
\hline 1 (b) & & $37(21-63)$ & Son (b) & & $51(20-78)$ \\
\hline $2(\mathrm{c})$ & & $51(22-76)$ & Sibling (c) & & $46(20-77)$ \\
\hline \multirow[t]{3}{*}{$3(d)$} & & $68(49-79)$ & Relative (d) & & $47,5(22-79)$ \\
\hline & $\mathrm{z}$ & 222,914 & & $\mathrm{z}$ & 12,342 \\
\hline & $\mathrm{p}$ & $<0,001$ & & $\mathrm{p}$ & 0,006 \\
\hline \multirow[t]{7}{*}{ Bonferroni \& Tamhane’s T2 p } & & & Bonferroni \& Tamhane's T2 p & & $b-a ; p=0,015$ \\
\hline & & $\mathrm{b}-\mathrm{a} ; \mathrm{p}<0,001$ & Caregiver Education & & \\
\hline & & $c-a ; p<0,001$ & Reading and writing (b) & & $48(20-77)$ \\
\hline & & $c-b ; p<0,001$ & Primary Education (c) & & $50(20-79)$ \\
\hline & & $\mathrm{d}-\mathrm{a} ; \mathrm{p}<0,001$ & High School and Above (d) & & $47(20-78)$ \\
\hline & & $d-b ; p<0,001$ & & $\mathrm{z}$ & 3,304 \\
\hline & & $d-c ; p<0,001$ & & $\mathrm{p}$ & 0,347 \\
\hline Patients Education & & & Bonferroni \& Tamhane's T2 p & & No Difference \\
\hline None (a) & & $59(26-78)$ & & & \\
\hline Reading and writing (b) & & $48(22-78)$ & & & \\
\hline Primary Education (c) & & $47,5(20-79)$ & & & \\
\hline \multirow[t]{3}{*}{ High School and Above(d) } & & $42,5(20-75)$ & & & \\
\hline & $\mathrm{z}$ & 17,885 & & & \\
\hline & $\mathrm{p}$ & $<0,001$ & & & \\
\hline \multicolumn{6}{|l|}{ Bonferroni \& Tamhane’s T2 p } \\
\hline & & $\mathrm{a}-\mathrm{d} ; \mathrm{p}<0,001$ & & & \\
\hline & & $b-d ; p=0,006$ & & & \\
\hline & & $c-d ; p=0,017$ & & & \\
\hline
\end{tabular}


The parameters found significant in assessing by the Zarit score and pairwise and more group analyses were researched via multivariate linear regression analysis (ENTER method). There was a statistically significant correlation between ECOG PS $(\mathrm{p}<0.001)$, transportation $(\mathrm{p}=0.018)$, residence status $(\mathrm{p}: 0.004)$, receiving chemotherapy $(\mathrm{p}<0.001)$, having a metastatic disease $(\mathrm{p}:<0.001)$ and the Zarit score $(\mathrm{p}<0.05$ for all $)$ (Table 3).

Statistically significant differences were found between the Zarit caregiver burden groups when the caregivers' Zarit load levels were assessed by SF 36 physical

Table 3. Zarit score linear regression analysis

\begin{tabular}{|c|c|c|c|c|c|}
\hline & \multicolumn{2}{|c|}{$\begin{array}{c}\text { Non- } \\
\text { Standardized } \\
\text { Coefficients }\end{array}$} & \multirow{2}{*}{$\begin{array}{c}\text { Standardized } \\
\text { Coefficients } \\
\text { Beta } \\
\end{array}$} & \multirow[t]{2}{*}{$\mathbf{t}$} & \multirow[t]{2}{*}{$\mathbf{p}$} \\
\hline & Beta & sd & & & \\
\hline $\begin{array}{l}\text { Patient } \\
\text { Comorbidity }\end{array}$ & 0.555 & 0.558 & 0.020 & 0.995 & 0.320 \\
\hline Ecog PS & 2.527 & 0.548 & 0.131 & 4.614 & $<0.001$ \\
\hline $\begin{array}{l}\text { Organ } \\
\text { Metastasis }\end{array}$ & 8.482 & 0.676 & 0.641 & 6.505 & $<0.001$ \\
\hline Chemotherapy & 3.486 & 0.746 & 0.236 & 5.752 & $<0.001$ \\
\hline $\begin{array}{l}\text { Patients } \\
\text { Education }\end{array}$ & -0.454 & 0.306 & -0.028 & -1.485 & 0.138 \\
\hline $\begin{array}{l}\text { Caregiver } \\
\text { Transportation }\end{array}$ & -0.150 & 0.075 & -0.116 & -1.985 & 0.018 \\
\hline $\begin{array}{l}\text { Caregiver Place } \\
\text { of Residence }\end{array}$ & -0.141 & 0.551 & -0.005 & -0.256 & 0.798 \\
\hline $\begin{array}{l}\text { Cargiver } \\
\text { Residence Status }\end{array}$ & 1.472 & 0.569 & 0.960 & 2.829 & 0.004 \\
\hline $\begin{array}{l}\text { Caregiver } \\
\text { İntimacy }\end{array}$ & -0.300 & 0.238 & -0.024 & -1.259 & 0.209 \\
\hline $\begin{array}{l}\text { Caregiver } \\
\text { Gender }\end{array}$ & -0.014 & 0.544 & -0.001 & -0.026 & 0.979 \\
\hline $\begin{array}{l}\text { Caregiver } \\
\text { Comorbidity }\end{array}$ & -0.188 & 0.602 & -0.006 & -0.313 & 0.755 \\
\hline
\end{tabular}

function and SF 36 physical role difficulty, SF 36 pain, SF 36 general health, SF 36 energy/vitality, SF 36 social functionality, SF 36 emotion, SF 36 mental health. Bonferroni \& Tamhane's test was performed to determine which subgroups caused the statistical difference between the groups. According to the test results, the source of the difference between caregivers' Zarit load level and SF 36 general health, SF 36 energy/ vitality, SF 36 social functionality, and SF 36 mental health scale scores; was determined that the caregivers without a load compared to the load level is medium and heavy, those with a light load level compared to with a medium and heavy load level, those with a medium load level compared to with a heavy load level had higher scores $(\mathrm{p}<0.05)$.

The cause of the difference between the groups assessing by Zarit load level and SF 36 physical function, SF 36 physical role difficulty scores; was observed that caregivers without burden compared to caregivers with a medium and heavy load, caregivers with light load levels compared to with a medium and heavy load had higher scores $(\mathrm{p}<0.05)$.

According to the SF 36 pain scale, it was stated that caregivers without a load compared to caregivers with a high load level, caregivers with a light load level compared to with a medium and heavy load level had higher scores $(\mathrm{p}<0.05)$.

Assessing of SF 36 emotional role difficulty scale, it was expressed that caregivers without a load compared to caregivers with a high load level, caregivers with a light load level compared to with a medium and heavy load level, caregivers with a medium load level compared to with a heavy load level had higher scores $(\mathrm{p}<0.05)$ (Table 4).

Table 4. Effects of Zarit caregiver burden score on Short Form 36

\begin{tabular}{|c|c|c|c|c|c|c|c|c|}
\hline & $\begin{array}{c}\text { SF36 } \\
\text { Physical } \\
\text { Function }\end{array}$ & $\begin{array}{c}\text { SF36 } \\
\text { Physical Role } \\
\text { Difficulty }\end{array}$ & SF36 Pain & $\begin{array}{c}\text { SF36 } \\
\text { General } \\
\text { Health }\end{array}$ & $\begin{array}{c}\text { SF36 } \\
\text { Energy / } \\
\text { Vitality }\end{array}$ & $\begin{array}{l}\text { SF36 Social } \\
\text { Functionality }\end{array}$ & $\begin{array}{c}\text { SF36 } \\
\text { Emotional } \\
\text { Role } \\
\text { Difficulty }\end{array}$ & $\begin{array}{l}\text { SF36 Mental } \\
\text { Health }\end{array}$ \\
\hline & $\begin{array}{c}\text { Med } \\
\text { (Min-Maks) }\end{array}$ & $\begin{array}{c}\text { Med } \\
\text { (Min-Max) }\end{array}$ & $\begin{array}{c}\text { Med } \\
\text { (Min-Max) }\end{array}$ & $\begin{array}{c}\text { Med } \\
(\text { Min-Max) }\end{array}$ & $\begin{array}{c}\text { Med } \\
\text { (Min-Max) }\end{array}$ & $\begin{array}{c}\text { Med } \\
\text { (Min-Max) }\end{array}$ & $\begin{array}{c}\text { Med } \\
\text { (Min-Max) }\end{array}$ & $\begin{array}{c}\text { Med } \\
\text { (Min-Max) }\end{array}$ \\
\hline \multicolumn{9}{|c|}{ Zarit Carrgiver Load Weight } \\
\hline None (a) & $54(46-77)$ & $56(44-68)$ & $56(41-65)$ & $57(40-70)$ & $51(40-73)$ & $59(49-77)$ & $59(39-64)$ & $63(50-66)$ \\
\hline Light Load (b) & $51(34-69)$ & $51(30-69)$ & $51(33-67)$ & $52(36-70)$ & $50(37-68)$ & $57(34-77)$ & $55(23-71)$ & $57(40-70)$ \\
\hline Medium Load (c) & $48(24-63)$ & $47(20-63)$ & $48(26-62)$ & $47(25-63)$ & $45(24-54)$ & $54(26-69)$ & $51(22-70)$ & $52(26-67)$ \\
\hline Heavy Load (d) & $32(20-50)$ & $31(12-58)$ & $38(22-59)$ & $38(24-60)$ & $39(23-53)$ & $48(24-65)$ & $35(20-58)$ & $45(24-60)$ \\
\hline $\mathrm{z}$ & 237,076 & 213,157 & 167,927 & 176,735 & 182,133 & 134,568 & 154,950 & 123,622 \\
\hline $\mathrm{p}$ & $<0,001$ & $<0,001$ & $<0,001$ & $<0,001$ & $<0,001$ & $<0,001$ & $<0,001$ & $<0,001$ \\
\hline \multicolumn{9}{|c|}{ Bonferroni \& Tamhane's T2 p } \\
\hline & $\mathrm{a}-\mathrm{c} ; \mathrm{p}<0,001$ & $a-c ; p=0,001$ & $\mathrm{a}-\mathrm{d} ; \mathrm{p}<0,001$ & $\mathrm{a}-\mathrm{c} ; \mathrm{p}=0,011$ & $\mathrm{a}-\mathrm{c} ; \mathrm{p}=0,002$ & $a-c ; p=0,002$ & $\mathrm{a}-\mathrm{d} ; \mathrm{p}<0,001$ & $\mathrm{a}-\mathrm{c} ; \mathrm{p}=0,017$ \\
\hline & $\mathrm{a}-\mathrm{d} ; \mathrm{p}<0,001$ & $\mathrm{a}-\mathrm{d} ; \mathrm{p}<0,001$ & $\mathrm{~b}-\mathrm{c} ; \mathrm{p}<0,001$ & $\mathrm{a}-\mathrm{d} ; \mathrm{p}<0,001$ & $\mathrm{a}-\mathrm{d} ; \mathrm{p}<0,001$ & $a-d ; p<0,001$ & b-c; $p<0,001$ & a-d; $p<0,001$ \\
\hline & b-c; $p<0,001$ & b-c; $p<0,001$ & b-d; $p<0,001$ & b-c; $p<0,001$ & b-c; $p<0,001$ & b-c; $p<0,001$ & b-d; $p<0,001$ & $\mathrm{~b}-\mathrm{c} ; \mathrm{p}=0,001$ \\
\hline & b-d; $p<0,001$ & b-d; $p<0,001$ & & b-d; $p<0,001$ & b-d; $p<0,001$ & b-d; $p<0,001$ & $\mathrm{c}-\mathrm{d} ; \mathrm{p}<0,001$ & b-d; $p<0,001$ \\
\hline & & & & $\mathrm{c}-\mathrm{d} ; \mathrm{p}<0,001$ & $\mathrm{c}-\mathrm{d} ; \mathrm{p}<0,001$ & $\mathrm{c}-\mathrm{d} ; \mathrm{p}<0,001$ & & $\mathrm{c}-\mathrm{d} ; \mathrm{p}<0,001$ \\
\hline
\end{tabular}


A moderate reverse relationship was detected between Zarit scores of caregiver $s$ and scale scores of SF36 physical function ( $\mathrm{r}=-0.744)$, SF 36 physical role difficulty $(\mathrm{r}=-0.697)$, SF 36 pain $(\mathrm{r}=-0.604)$, SF 36 general health $(r=-0.624)$, SF 36 energy / vitality $(r=-0.635)$, SF 36 social functionality $(\mathrm{r}=-0.545)$, SF 36 emotional role difficulty $(\mathrm{r}=-0.575)$, SF 36 mental health $(\mathrm{r}=-0.535) \quad(\mathrm{p}<0.05)$ (Table 5).

\begin{tabular}{|lcc|}
\hline \multicolumn{3}{|c|}{ Table 5. Zarit caregiver burden and Short form 36 correlation } \\
\hline & Zarit & p \\
\hline SF36Physical function & r & $<0,001$ \\
SF36Physical Role Difficulty & $-0,744^{* *}$ & $<0,001$ \\
Sf36 Pain & $-0,697$ & $<0,001$ \\
SF36General Health & $-0,604$ & $<0,001$ \\
SF36Energy /Vitality & $-0,624$ & $<0,001$ \\
SF36Social Functionality & $-0,635$ & $<0,001$ \\
SF36Emotional Role Difficulty & $-0,545$ & $<0,001$ \\
SF36 Mental Healt & $-0,575$ & $<0,001$ \\
\hline * $<$ 00,05, Spearman correlation test & $-0,535$ & \\
\hline
\end{tabular}

\section{DISCUSSION}

A multiple evaluation strategy was applied in our study. The effects of the clinical characteristics of 411 patients who were followed up and treated in our hospital's oncology polyclinics between January 2020 and March 2021 and the social status of their caregivers on Zarit caregiver burden were perused. In addition, the effects of Zarit caregiver burden on the short form 36, which indicates the quality of life of the caregiver, were researched.

It was confirmed that PS, patient education level, age, gender, having additional comorbidity, having metastatic disease, receiving chemotherapy, using public transportation, village or district residence, becoming tenants, gender were associated with high Zarit caregiver score in our study $(\mathrm{p}<0.05$ for all).

It was detected that PS, using public transportation, becoming tenant, receiving chemotherapy, having metastatic disease were associated with a high Zarit caregiver burden score in the multivariate linear regression analysis $(\mathrm{p}<0.05$ for all).

It was determined that when the Zarit caregiver burden score increase, SF 36 scores of the caregiver's quality of life scale score decrease $(\mathrm{p}<0.05$ for all).

The rise in chronic diseases with the aging population and the promising treatment methods that have emerged in the last century in treating chronic diseases reveals a patient population in need of long-term care. The patient group with cancer has an essential part in this population. Some of the patients are influenced in a wide range from physical independence to complete dependence because of the physical effect of cancer on patients and/or the life quality impairment in patients receiving cancer treatment. The majority of patients need continuous daily care. Unfortunately, this need is often met by family members or close friends who do not have the qualifications and training in care for the vast majority. While people who undertake caring for patients struggle with problems they have no experience with, they endeavor to go on their lives to cope with their health problems and stress. Difficulty in dealing with more than one problem makes caregivers feel insufficient, so this situation negatively affects the caregiver's quality of life as much as the patient $(16,17)$.

One of the quick and elementary indicators of whether or the patient can survive without support is the performance score evaluation. It was reported that caregivers of patients with PS:3-4 had a statistically significantly higher Zarit caregiver burden score compared to caregivers of patients with PS:0-2 in a study of patients with lung cancer about performance score (PS) and Zarit caregiver burden by Wood et al. (18) (p:0.008). It was stated that high Zarit caregiver burden scores were observed in caregivers of patients with high-performance scores in the study on 441 cancer patients aged 60-80 years by Semere et al. (19). It was revealed that poor performance score was a factor predicting high Zarit caregiver burden in the study on patients with brain tumors by Bayen et al. (20). PS was demonstrated as one of the main factors affecting Zarit caregiver burden in line with the general literature in multivariate linear regression analysis in our $\operatorname{study}(\mathrm{p}<0.005)$.

Studies focusing on cancer's financial burden on the caregiverr express a heavy economic burden on the patient and their caregivers, additionally the physical and psychological burden. It was demonstrated that informal caregiver expenditure costs $\$ 4563$ for each patient per three-month average; however, this expense was not less than nursing homes and official patient care institutions in a study on cancer patients by Stommel et al. (21). It was found that total informal expenditure was average out $\$ 7,290$ for three months, and caregivers of patients in the last six months of their lives had more expense in another study that specifically examined the costs of adult cancer care (22). The annual cost of the disease to caregivers was estimated as 7,028, 19,701, and 14,234 dollars in the first year, after the first year, and in the terminal period, respectively, in a study on caregivers of patients with colorectal and lung cancer by Van Houtven et al. (23). Longo et al. also reported that $20 \%$ of cancer patients' caregivers experience significant financial problems (24). We speculated that patients and caregivers with low socioeconomic status would be exposed to higher 
caregiver burden due to the additional financial burden. Consequently, it was determined that parameters that were questioned and taken as indicators of low economic status in our study, such as using public transportation and becoming tenant, were associated with a higher caregiver burden ( $\mathrm{p}<0,05$ for all).

The physical and psychological conditions of cancer patients become worse, usually during chemotherapy treatment. When the duration of chemotherapy increases, it is possible to develop functional impairment due to emerged and increased adverse effects (25). Correlatively, it has been reported that caregivers of cancer patients experience intense mental problems during the diagnosis and treatment of cancer, and social functionality is negatively affected (26). It was specified that caregivers have difficulties managing adverse effects and have concerns about the effectiveness of the treatment during the cancer treatment of patients in the study performed by Northouse et al. (27). Inadequacy coping skills because of the adverse effects of developing in patients during chemotherapy and the difficulties managing these adverse effects may explain having more significant caregivers' burden during chemotherapy (28). Our study showed that caregivers of patients receiving active chemotherapy treatment had higher Zarit caregiver burden scores than caregivers of cancer patients who were not receiving chemotherapy treatment $(\mathrm{p}<0.05)$.

Metastatic cancer patients may have many difficult to manage symptoms; besides, these patients are likely to receive aggressive treatments and are often exposed to a considerable amount of treatment-related adverse effects (29). It has been shown that caregivers of metastatic cancer patients have more intensive anxiety symptoms by some authors (30-31). It was demonstrated that caregivers of patients with relapsed and metastatic disease have more distress, strain, and compliance problems than caregivers of patients with early-stage disease in a study performed by Morse et al. (32). The findings in our research that caregivers of metastatic cancer patients had a higher Zarit caregiver score than caregivers of patients without metastases supports these results $(\mathrm{p}<0.05)$.

Many studies have focused on the life quality of cancer patients during diagnosis and treatment. The studies about the life quality of cancer patients' caregiver s are relatively limited in the literature $(33,34)$. The lower SF36 scores were obtained from the caregivers included in the study in mental health, vitality, and social functioning subgroups compared to the normal population in a study performed on 96 individuals caring for cancer patients by Grov et al. (35). It was determined that pain and mental role were the most affected SF-36 subscales in a study on caregivers of patients with hematological malignancies by Gereklioglu et al. (36). Our study is the first to compare Zarit caregiver burden score with SF-36 scores on caregivers of cancer patients in the literature review. Our results represent that SF-36 scores, one of the life quality scales, decrease in conjunction with Zarit caregiver burden increases in caregivers. There is a moderate reverse relationship between Zarit scores and SF36 subscales. It can be deduced from the results of our study that social support services for patients and their caregivers should be used more effectively and intensively to maintain the well-being of the patient, although the state pays for oncological treatments in many countries

There are some limitations in our study. The most important limitations of our study are that it was conducted in a single-center, cross-sectional, and limited population, which is a homogeneous community with a specific culture. However, we think our study as the first study to compare the Zarit caregiver burden questionnaire and the SF-36 questionnaire on caregivers will contribute to the literatüre..

\section{CONCLUSION}

Nowadays, only disease-oriented medicine practices are unfortunately insufficient to evaluate the patient's well-being in a physically, psychologically, socially, and economically devastating disease such as cancer. Inadequate social support usually causes treatment incompatibility, which means shorter disease-free and overall survival for patients. Therefore, we think that the caregivers of cancer patients are also an essential factor that should be considered in terms of the patient's well-being and treatment compliance. Our study reveals the need to peruse the patient with the caregiver as a whole, although caregivers are ignored during medical practices. Zarit caregiver burden scores are influenced by the patient's clinical characteristics and the caregiver's social and economic status. The increased Zarit caregiver burden negatively affects the caregiver's life quality.

\section{ETHICAL DECLARATIONS}

Ethics Committee Approval: This study received approval from the Institutional Review Board of Health Sciences University Diyarbakır Gazi Yaşargil Training and Research Hospital Ethics Committee (Date 09.06.2021; Decision No: 829).

Informed Consent: All patients signed the free and informed consent form.

Referee Evaluation Process: Externally peer-reviewed. Conflict of Interest Statement: The authors have no conflicts of interest to declare.

Financial Disclosure: The authors declared that this study has received no financial support. 
Author Contributions: All of the authors declare that they have all participated in the design, execution, and analysis of the paper, and that they have approved the final version.

\section{REFERENCES}

1. Hyuna S, Jacques F,Rebecca L, et al. Global cancer statistics 2020: GLOBOCAN estimates of incidence and mortality worldwide for 36 cancers in 185 countries. Ca Cancer J Clin 2021; 0: 1-41.

2. Christopher J L Murray. Global, regional, and national age-sex specific mortality for 264 causes of death, 1980-2016: a systematic analysis for the Global Burden of Disease Study 2016. Lancet 2017; 390: 1151-210.

3. Blum K, Sherman DW. Understanding the experience of caregivers: a focus on transitions. Semin Oncol Nurs 2010; 26: 243-58.

4. NAC [Accessed 01/03/20]; Caregiving in the U.S. 2020 https:// www.caregiving.org/ caregiving-in-the-us-2020/

5. Lee S, Colditz GA, Berkman LF, Kawachi I. Caregiving and risk of coronary heart disease in U.S. women: A prospective study. Am J Prev Med 2003; 24: 113-9.

6. Schulz R, Beach SR. Caregiving as a risk factor for mortality - The caregiver health effects study. Jama-J Am Med Assoc 1999; 282: 2215-9.

7. Buyan N, Türkmen MA, Bilge I, et al. Quality of life in children with chronic kidney disease (with child and parent assessments). Pediatr Nephrol 2010; 25: 1487-96.

8. Rahmani F, Ranjbar F, Hosseinzadeh M, et al. Coping strategies of family caregivers of patients with schizophrenia in Iran: A crosssectional survey. Int J Nurs Sci 2019; 6: 148-53.

9. Mora-Castañeda B, Márquez-González M, Fernández-Liria A, et al. Clinical and demographic variables associated coping and the burden of caregivers of schizophrenia patients. Rev Colomb Psiquiatr 2016; 47: 13-20.

10. Parekh NK, Shah S, McMaster K, et al. Effects of caregiver burden on quality of life and coping strategies utilized by caregivers of adult patients with inflammatory bowel disease. Ann Gastroenterol 2017; 30: 89-95.

11.Zarit SH, Reever KE, Bach-Peterson J. Relatives of the impaired elderly: correlates of feeling of burden. Gerontologist 1980; 20: 649-55

12. Özlü A, Yıldız M, Aker T. A reliability and validity study on the Zarit caregiver burden scale. Archives of Neuropsychiatry 2009; 46: $38-42$

13. Ware JE, Sherbourne CD. “The MOS 36-item Short Form Healty Survey”, I. Conceptual Framework and item Selection, Med Care, 1992.

14. Bowling A. "Measuring Health: A Review of Quality of Life Measurement Scales", II. En. Open Univesity Press, Philadelphia, 1997.

15. Koçyiğit H, Aydemir Ö, Fisek G, et al. "Reliability and Validity of the Turkish Version of Short Form-36", Journal of Medicine and Treatment, 1999.

16. O'Hara RE, Hull JG, Lyons KD, et al. Impact on caregiver burden of a patient-focused palliative care intervention for patients with advanced cancer. Palliat Support Care 2010; 8: 395-404.

17. Harding R, Higginson IJ, Donaldson N. The relationship between patient characteristics and carer psychological status in home palliative cancer care. Support Care Cancer 2010; 11: 638-43.

18. Wood R, Taylor-Stokes G, Smith F et al. The humanistic burden of advanced non-small cell lung cancer: What are the key drivers of caregiver burden? J Clin Oncol 2018; 36: 149-149.
19. Semere W, D.Althouse A, Rosland A.M et al. Poor patient health is associated with higher caregiver burden for older adults with advanced cancer Poor patient health is associated with higher caregiver burden for older adults with advanced cancer. J Geriatr Oncol 2021.doi.org/10.1016/j. jgo.2021.01.002.

20.Bayen E, Donadey FL, Prouté M, et al. The multidimensional burden of informal caregivers in primary malignant brain tumor. Supportive Care in Cancer 2017; 25: 245-53.

21. Stommel M, Given CW, Given BA. The cost of cancer home care to families Cancer 1993; 71: 1867-74.

22. Given BA, Given CW, Stommel M. Family and out-of-pocket costs for women with breast cancer. Cancer Pract 1994; 2: 187-93.

23. Van Houtven $\mathrm{CH}$, Ramsey SD, Hornbrook MC, et al. Economic burden for informal caregivers of lung and colorectal cancer patients. The Oncologist 2010; 15: 883- 93.

24. Longo CI, Fitch M, Deber RB, Williams AP. Financial and family burden associated with cancer treatment in Ontario, Canada. Supportive Care in Cancer 2006; 14: 1077-85.

25. Almohammadi A, Alqarni A, Alraddadi R, et al. Assessment of patients' knowledge in managing side effects of chemotherapy: case of King Abdul-Aziz University Hospital. J Cancer Educ 2020; 35: $334-8$

26. Traa MJ, Braeken J, De Vries J, Roukema JA, Orsini RG, Den Oudsten BL. Evaluating quality of life and response shift from a couple-based perspective: a study among patients with colorectal cancer and their partners. Qual Life Res 2015; 24: 1431-41.

27. Northouse LL, Katapodi MC, Schafenacker AM, Weiss D. The impact of caregiving on the psychological well-being of family caregivers and cancer patients. Semin Oncol Nurs 2012; 28: 23645.

28. Palacio C, Krikorian A, Limonero JT. The influence of psychological factors on the burden of caregivers of patients with advanced cancer: resiliency and caregiver burden. Palliat Support Care 2018; 16: 269-77.

29. Given BA, Given CW, Kozachik S, et al. Family support in advanced cancer. J Cancer Clinicians 2001; 51: 4.

30.Ellis J. The impact of lung cancer on patients and carers. Chron Respir Dis 2012; 9: 39-47.

31. Burridge LH, Barnett AG, Clavarino AM. The impact of perceived stage of cancer on carers' anxiety and depression during the patients' final year of life. Psychooncology 2009; 18: 615-23.

32. Morse SR, Fife B. Coping with a partner'scancer: adjustment at four stages of the illness tra-jectory. Oncol Nurs Forum 1998; 25: 751-60.

33. Grov EK, Dahl AA, Moum T, Foss SD. Anxiety, depression, and quality of life in caregivers of patients with cancer in late palliative phase Ann Oncol 2005; 16: 1185-91.

34. Wagner CD, Bigatti SM, Storniolo AM. Quality of life of husbands of women with breast cancer. Psychooncology 2006; 15: 109-20.

35. Grov EK, Dahl AA, Moum T, Fosså SD. Anxiety, depression, and quality of life in caregivers of patients with cancer in late palliative phase. Ann Oncol 2005; 16: 1185-91.

36. Gereklioğlu Ç, Korur A, Asma S, et al. Assessment of quality of life in caregivers of the patients with hematologic neoplasms. Turk J Fam Med Primary Care 2019; 13: 3. 